\title{
Diagnosis and treatment of epididymal tuberculosis: A review of 47 cases
}

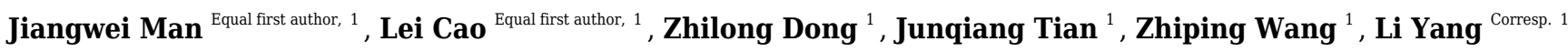 \\ ${ }^{1}$ Department of Urology, Lanzhou University Second Hospital, Lan Zhou, China \\ Corresponding Author: Li Yang \\ Email address: ery_yangli@|zu.edu.cn
}

Objective: To analyze the clinical manifestations, diagnosis, and treatment outcomes in a series of patients with epididymal tuberculosis. Methods: This study is a retrospective data analysis of 47 cases of histologically-confirmed epididymal tuberculosis in patients treated at our hospital from November 2012 to December 2018.

Results: The average age of the patients was approximately 42 years. The epididymal lesion location was left-sided in 15 patients(31.9\%), right-sided in 22 patients (46.8\%), and bilateral in 10 patients $(21.3 \%)$. The main symptoms were painless swelling of the scrotum in 21 cases $(44.7 \%)$ and scrotal drop pain in 21 cases $(44.7 \%)$. Scrotal physical examination revealed epididymal beaded enlargement in 12 patients (25.5\%), testicular mass in 1 patient $(2.1 \%)$, scrotal tenderness alone in 7 patients (14.9\%), illdefined epididymal-testicular border in 21 patients (44.7\%), and sinus formation in 6 patients (12.8\%). After 2 to 4 weeks of anti-tuberculosis chemotherapy, the patients underwent a surgical procedure. We found that 10 (83.3\%) of the 12 patients whose main symptom was epididymal beaded enlargement underwent simple epididymal surgery. Of the 21 patients whose main clinical manifestation was illdefined testis-epididymis demarcation, 16 (72.2\%) underwent epididymis-testicular surgery. All patients underwent postoperative chemotherapy for 3 to 6 months. Postoperative follow-up showed good response to treatment.

Conclusion: It is difficult to diagnose early-stage epididymal tuberculosis. Epididymal tuberculosis is likely to have invaded surrounding tissues when signs such as epididymal beaded changes and ill-defined epididymis-testis border are present. Surgical treatment combined with preoperative and postoperative chemotherapy is an effective approach to treating this condition. 
1 Diagnosis and treatment of epididymal tuberculosis: A review of 47 cases

2 Jiangwei Man*, Lei Cao*, Zhilong Dong, Junqiang Tian,Zhiping Wang, Li Yang

3

Department of Urology, Lanzhou University Second Hospital, Lan Zhou,China

* These authors contributed equally to this work

6 Corresponding Author: Li Yang

7 Email address: ery_yangli@lzu.edu.cn

\section{Abstract:}

Objective: To analyze the clinical manifestations, diagnosis, and treatment outcomes in a series of patients with epididymal tuberculosis.

Methods: This study is a retrospective data analysis of 47 cases of histologically-confirmed epididymal tuberculosis in patients treated at our hospital from November 2012 to December 2018.

Results: The average age of the patients was approximately 42 years. The epididymal lesion location was left-sided in 15 patients $(31.9 \%)$, right-sided in 22 patients $(46.8 \%)$, and bilateral in 10 patients $(21.3 \%)$. The main symptoms were painless swelling of the scrotum in 21 cases (44.7\%) and scrotal drop pain in 21 cases (44.7\%). Scrotal physical examination revealed epididymal beaded enlargement in 12 patients $(25.5 \%)$, testicular mass in 1 patient $(2.1 \%)$, scrotal tenderness alone in 7 patients (14.9\%), ill-defined epididymal-testicular border in 21 patients (44.7\%), and sinus formation in 6 patients (12.8\%). After 2 to 4 weeks of anti-tuberculosis chemotherapy, the patients underwent a surgical procedure. We found that $10(83.3 \%)$ of the 12 patients whose main symptom was epididymal beaded enlargement underwent simple epididymal surgery. Of the 21 patients whose main clinical manifestation was ill-defined testis-epididymis demarcation, 16 (72.2\%) underwent epididymis-testicular surgery. All patients underwent postoperative chemotherapy for 3 to 6 months. Postoperative follow-up showed good response to treatment.

Conclusion: It is difficult to diagnose early-stage epididymal tuberculosis. Epididymal tuberculosis is likely to have invaded surrounding tissues when signs such as epididymal beaded changes and ill-defined epididymis-testis border are present. Surgical treatment combined with preoperative and postoperative chemotherapy is an effective approach to treating this condition.

Keywords: epididymal tuberculosis, clinical characteristics, chemotherapy

Introduction: Epididymal tuberculosis is a rare extrapulmonary form of tuberculosis that occurs in young adults(Surati et al. 2012). Patients with this disease may have no obvious clinical 
symptoms or only mild symptoms. The disease typically develops slowly and early diagnosis is difficult; delayed diagnosis and misdiagnosis are common. Recently, due to the emergence of multi-drug resistant bacteria (MDR), anti-tuberculosis drug resistance, and the widespread use of glucocorticoids, the incidence of Male genital TB, including epididymal tuberculosis, has been increasing worldwide. Epididymal tuberculosis remains an important health problem in many developing countries, including China(Kulchavenya et al. 2012). The pathological features of epididymal tuberculosis are extensive tissue destruction and fibrosis, eventually leading to destruction of the epididymis and surrounding genital tissues and organs and complications such as infertility and other serious effects on male reproductive system function.

At present, early diagnosis and regular chemotherapy are the keys to cure male reproductive system tuberculosis and to avoid surgical treatment. However, due to its late onset and nonspecific clinical signs and symptoms, and the lack of rapid, sensitive, and specific diagnostic methods, the disease is often misdiagnosed or the diagnosis is delayed. Therefore, surgical treatment combined with chemotherapy has been the preferred treatment approach to this disease. This study, a retrospective analysis of a series of cases of epididymal tuberculosis, is intended to more fully characterize the clinical features and outcomes of surgical treatment of this disease.

Material and Methods: We collected the medical records of 47 patients with histologicallydiagnosed epididymal tuberculosis from November 2012 to October 2018 at the Second Hospital of Lanzhou University. Age, clinical signs and symptoms, diagnostic methods, and treatment effects were collected from the patients' electronic medical records, and the final results were compared and analyzed. The following information was supplied relating to ethical approvals (i.e., approving body and any reference numbers): Ethics Committee of Lanzhou University Second Hospital Number: 2019A-079. Written consent was obtained from all patients.

Results: The mean age of the patients was 41.98 years (range, 19-72 years); 15 patients (31.9\%) had tuberculosis in the left epididymis, 22 patients (46.8\%) had tuberculosis in the right epididymis, and 10 patients $(21.3 \%)$ had bilateral epididymal tuberculosis. Of the 47 patients, 8 (17.0\%) had a history of tuberculosis, including 3 cases of pulmonary tuberculosis, 4 cases of renal tuberculosis, and 1 case of prostate tuberculosis ( Table 1).

Main symptoms at the time of onset included: painless swelling of the scrotum in 21 patients (44.7\%); scrotal drop pain in 21 patients (44.7\%); urinary tract irritation such as urinary frequency, dysuria, and hematuria in 4 patients $(8.5 \%)$; and scrotal skin ulceration in 1 patient $(2.1 \%)$. Systemic symptoms such as low-grade fever, fatigue, and night sweats occurred in 6 patients $(12.8 \%)$. All patients underwent scrotal physical examination, which revealed epididymal beaded enlargement in 12 patients $(25.5 \%)$, testicular mass in 1 patient $(2.1 \%)$, scrotal tenderness alone in 7 patients (14.9\%), ill-defined epididymal testicular border in 21 patients $(44.7 \%)$, and sinus formation in 6 patients $(12.8 \%)$ ( Table 1).

Urinalysis and chest imaging were performed in all patients. Twenty patients $(42.6 \%)$ were positive for white blood cells in urine and 8 patients (17.0\%) were positive for red blood cells in 
72 urine. Chest imaging was positive in 8 patients $(17.0 \%)$. The preoperative diagnoses were epididymal tuberculosis in 41 patients $(87.2 \%)$, epididymal tumor in 3 patients $(6.4 \%)$, and epididymal mass in 3 patients (6.4\%). All patients had evidence of surgical treatment of the lesion.

Among the 47 cases, $41(87.2 \%)$ were initially identified as epididymal tuberculosis and treated pre-operatively with rifampicin, isoniazid, pyrazinamide, and ethambutol for 2 to 4 weeks. 26 patients $(55.3 \%)$ had epididymectomy and 21 patients $(44.7 \%)$ had epididymectomy combined with orchiectomy, and all recovered well after surgery.

We found that $10(83.3 \%)$ of the 12 patients whose main symptom was epididymal beaded enlargement underwent simple epididymal surgery and 2 (16.7\%) underwent epididymis-testicular surgery. Of the 21 patients whose main clinical manifestation was ill-defined testis-epididymis demarcation, $16(72.2 \%)$ underwent epididymis-testicular surgery and 5 epididymal surgery ( Table 2 ).

Macroscopically, all specimens contained solitary or confluent pale, grayish caseous necrotic nodules. Some lesions invaded the entire epididymis, some showed involvement of the testis with adherence to the scrotum to form a cold abscess, and some showed the formation of ulcerated sinus tracts in the skin. Microscopically, the centers of the specimens were red-stained with amorphous granular, irregularly sized foci of caseous necrosis surrounded by tuberculous granulation tissue (epithelioid cells, Langhans giant cells, and lymphocytes) (Figure 1,2).

All patients received anti-tuberculosis treatment with rifampicin, isoniazid, pyrazinamide, or ethambutol after surgery for 3 to 6 months. No recurrence was reported.

\section{Discussion :}

The number of TB patients in China accounted for $10 \%-12 \%$ of the total number of TB patients in the world, ranking second in the world(Glaziou et al. 2018; Zhang et al. 2016). People in Gansu Province of China have a high incidence of tuberculosis and are relatively delayed in seeking medical care(Zhang et al. 2016). The presentation of tuberculosis is increasingly atypical. Unfortunately, the prevalence of drug-resistant strains of TB is increasing(Lee et al. 2015). The main treatment of epididymal tuberculosis is early anti-tuberculosis treatment. However, since most of the patients with epididymal tuberculosis in Gansu are in the terminal stage, surgical treatment combined with chemotherapy has been the best treatment modality for this type of patients.

Reproductive system tuberculosis can occur in any age, mainly in men 30 to 50 years old. Due to the long incubation period, it is not common in children. The most commonly involved organ is the epididymis, followed by the seminal vesicle, prostate, testis, and vas deferens(Yadav et al. 2017). Isolated epididymal tuberculosis is very rare(Gueye et al. 1998). One new study indicated that isolated epididymal tuberculosis may be the first or only manifestation of early genitourinary tuberculosis(Viswaroop et al. 2005). Similarly, in our patient cohort,there were 39 cases (82.9\%) 
108

109

110

111

112

113

114

115

116

117

118

119

120

121

122

123

124

125

126

127

128

129

130

131

132

133

134

135

136

137

138

139

140

141

142

143

144

of isolated epididymal tuberculosis.

The pathogenesis of epididymal tuberculosis includes blood-borne transmission and transurethral reflux of Mycobacterium tuberculosis caused by factors such as trauma, alcohol abuse and excessive sexual activity(Tzvetkov \& Tzvetkova 2006). Epididymal tuberculosis lesions first appear in the tail of the epididymis, owing to its rich blood supply and to retrograde infection from the vas deferens. The lesions of epididymal tuberculosis gradually invade the body to the head, finally affecting the entire epididymis. In severe cases, the testis can be involved(Chung et al. 1997). In this group, 21 patients $(44.7 \%)$ with epididymal tuberculosis invading the testis underwent radical surgery of the testicle. In our study, the main cause of the patient's visit was painless mass of the epididymis, which is consistent with previous studies(Kho \& Chan 2012). However, the proportion of patient with scrotal pain is higher, mainly because the patients included in this study all had advanced epididymal tuberculosis or involvement of the testis or scrotum.

The gold standard for diagnosing tuberculosis is the isolation and culture of Mycobacterium tuberculosis. In cases of suspected Male genital TB, we usually look for M. tuberculosis in the urine or tissue.Traditionally, the appearance of so-called sterile pyuria on microscopic urinalysis is considered to be a typical manifestation of urogenital involvement. Some finds reported that leukocytes in urine were present microscopically or grossly in a majority of cases $(50 \%$ and $10 \%$, respectively). Hematuria is a common symptom of urinary tuberculosis, which is mainly caused by renal tuberculosis and bladder tuberculosis Hematuria and acidic urine have been associated with urinary tuberculosis, but they are nonspecific findings. In our patient cohort $17.0 \%$ were positive for red blood cells in urine, which is related to renal tuberculosis.Leukocyte positivity in the urine contributed to the diagnosis in $20(42.6 \%)$ patients, but its specificity was low. Color Doppler ultrasound is the first choice for imaging analysis of epididymal tuberculosis(Viswaroop et al. 2005). CT and MR have little value in diagnosing epididymal tuberculosis; they are mainly used to diagnose tuberculosis in lung and kidney and provide support for the diagnosis of epididymal tuberculosis. Epididymal fine needle biopsy is a good method for the diagnosis of epididymal tuberculosis.

Tuberculous epididymitis can be the only manifestation of genitourinary tuberculosis. Therefore, even in the absence of clinical and laboratory markers of renal and urologic tuberculosis, all men with identifed epididymal lesions should undergo a fine needle aspiration biopsy. Kim(Kim et al. 1993)suggested that epididymal tuberculosis can often be diagnosed by Bultrasound biopsy, which supports the above conclusions. Polymerase chain reaction has been an important method for diagnose. Combined with pathological biopsy, it can improve the diagnosis rating epididymal tuberculosis in recent years(Chawla et al. 2012). It has the characteristics of high sensitivity, high specificity, and short turnaround tie. However, at present, the diagnosis of simple epididymal tuberculosis is difficult, and there is no preoperative diagnostic method with high sensitivity and specificity. The clinical diagnosis of scrotal abscess and ulceration is not difficult. 
It is often possible to make a clear diagnosis by examining for acid-fast bacilli in samples of the ruptured tissue through pus or secretion smears.

The differential diagnosis of epididymal tuberculosis includes bacterial epididymitis, epididymal sperm granuloma, epididymal tumor, and other diseases. Of the 47 patients, 41 had typical symptoms, signs, imaging findings or a history of tuberculosis, and they could be diagnosed with epididymal tuberculosis. 3 patients had no typical symptoms of tuberculosis and were misdiagnosed as epididymal masses because of the characteristics of the tumor (30-50 years, weight loss, solid epididymal mass). 3 patients without the above symptoms required surgery because of the mass affecting life. Patients with bacterial epididymitis often have testicularepididymis pain, and scrotal swelling and heat(Banyra \& Shulyak 2012). Features include a shorter course of illness, and symptoms are generally relieved after antibiotic treatment. Carl(Carl \& Stark 1997) reported that epididymal tuberculosis should be highly suspected when patients have persistent or repeated epididymitis episodes and symptoms cannot be controlled after adequate antibiotic treatment.

Epididymal sperm granuloma mainly occurs in the head of the epididymis, characterized by a smooth solid mass, and antibiotics and anti-tuberculosis treatment are ineffective. Epididymal tumors are rare, accounting for about $0.9 \%$ of male reproductive system tumors. Most tumors occur during periods of sexual activity in men between 20 and 40 years of age. The disease is characterized by slow growth, large volume, and no tenderness. CT helps to confirm the diagnosis.

Epididymal tuberculosis, like other tuberculosis diseases, requires early, regular, full-course, moderate, combined anti-tuberculosis treatment. The drug treatment method uses 3 to 4 antituberculosis drugs for 6 to 9 months(Peloquin 2014). Surgical treatment is necessary if there is no response to drug treatment or in cases of abscess formation. Because the early symptoms of epididymal tuberculosis are not obvious, abscesses or involvement of surrounding tissues such as the testicles have often developed at the time of treatment, so most patients need surgery. When there is active tuberculosis, anti-tuberculosis treatment must be performed before surgical treatment. In this study, Chest imaging of 8 patients (17.0\%) suggested that pulmonary tuberculosis had developed calcification, which proved that they were old tuberculosis. 41 (87.2\%) were identified as epididymal tuberculosis and were given antituberculous therapy for 2 to 4 weeks before surgery. In 28 cases in our patient cohort (59.6\%) presenting as a testicular painless mass, the epididymal-testis border was unclear, or sinus formation was found, suggesting that the surrounding tissues were invaded. Although surgical treatment is effective, patients still need regular anti-tuberculosis chemotherapy for 3 to 6 months after surgery and close follow-up.

Conclusion: epididymal tuberculosis is easy to diagnose in patients with a history of tuberculosis. However, in isolated epididymal tuberculosis, early symptoms are not obvious and cases are typically advanced at the time of diagnosis. There are obvious symptoms and signs such as epididymal enlargement, falling pain and bead-like changes. Epididymal tuberculosis has invaded 
182 surrounding tissue by the time it is discovered. Surgical treatment combined with preoperative and 183 postoperative chemotherapy is an effective treatment approach. When epididymal tuberculosis

184

185

186

187

188

189

190

191 manifests bead-like changes, testicular involvement, or sinus formation, good results can be achieved with definitive epididymal-testicular surgery. This experiment involved a relatively small number of cases, and the patients did not undergo needle biopsy. Therefore, further research is needed to further characterize this disease and refine the treatment.

\section{Reference}

Banyra O, and Shulyak A. 2012. Acute epididymo-orchitis: staging and treatment. Cent European J Urol 65:139-143.

Carl P, and Stark L. 1997. Indications for Surgical Management of Genitourinary Tuberculosis. World Journal of Surgery 21:505-510.

Chawla A, Chawla K, Reddy S, Arora N, Bairy I, Rao S, Hegde P, and Thomas J. 2012. Can Tissue PCR Augment the Diagnostic Accuracy in Genitourinary Tract Tuberculosis? Urologia Internationalis 88:34-38.

Chung JJ, Kim MJ, Lee T, Yoo HS, and Lee JT. 1997. Sonographic findings in tuberculous epididymitis and epididymoorchitis. J Clin Ultrasound 25:390-394.

Glaziou P, Floyd K, and Raviglione MC. 2018. Global Epidemiology of Tuberculosis. Semin Respir Crit Care Med 39:271285. $10.1055 / \mathrm{s}-0038-1651492$

Gueye SM, Ba M, Sylla C, Ndoye A, Fall A, Diaw J, and Mensah A. 1998. Epididymal manifestations of urogenital tuberculosis. Progrès En Urologie Journal De Lassociation Française Durologie Et De La Société Française Durologie 8:240.

Kho KS, and Chan PH. 2012. Isolated tuberculous epididymitis presenting as a painless scrotal tumor. Journal of the Chinese Medical Association Jcma 75:292-295.

Kim SH, Pollack HM, Cho KS, Pollack MS, and Han MC. 1993. Tuberculous Epididymitis and Epididymo-Orchitis: Sonographic Findings. J Urol 150:81-84.

Kulchavenya E, Kim C-S, Bulanova O, and Zhukova I. 2012. Male genital tuberculosis: epidemiology and diagnostic. World Journal of Urology 30:15-21.

Lee HY, Lee J, Lee YS, Kim MY, Lee HK, Lee YM, Shin JH, and Ko Y. 2015. Drug-resistance pattern of Mycobacterium tuberculosis strains from patients with pulmonary and extrapulmonary tuberculosis during 2006 to 2013 in a Korean tertiary medical center. Korean J Intern Med 30:325-334. 10.3904/kjim.2015.30.3.325

Peloquin DCA. 2014. Therapeutic Drug Monitoring in the Treatment of Tuberculosis. Drugs 74:839-854.

Surati KN, Suthar KD, and Shah JK. 2012. Isolated Tuberculous Epididymo-Orchitis: A Rare and Instructive Case Report. Southeast Asian Journal of Case Report \& Review.

Peer) reviewing PDF | (2019:08:40041:1:2:CHECK 7 Nov 2019) 
214 Tzvetkov D, and Tzvetkova P. 2006. TUBERCULOSIS OF MALE GENITAL SYSTEM - MYTH OR REALITY IN 21ST CENTURY. Archives of Andrology 52:375-381.

Viswaroop BS, Kekre N, and Gopalakrishnan G. 2005. Isolated tuberculous epididymitis: a review of forty cases. Journal of Postgraduate Medicine 51:109.

Yadav S, Singh P, Hemal A, and Kumar R. 2017. Genital tuberculosis: current status of diagnosis and management. Translational Andrology \& Urology 6:222-233. nephrectomy for renal tuberculosis: a retrospective study of 120 patients. Peerj 4:e2708. 


\section{Table $\mathbf{1}$ (on next page)}

\section{Table 1}

The clinical manifestations and diagnosis in 47 patients with epididymal Tuberculosis. 


\begin{tabular}{|c|c|c|}
\hline & number & $\begin{array}{l}\text { percentage } \\
(\%)\end{array}$ \\
\hline \multicolumn{3}{|l|}{ Location } \\
\hline left & 15 & 31.90 \\
\hline right & 22 & 46.80 \\
\hline bilateral & 10 & 21.30 \\
\hline \multicolumn{3}{|l|}{ Symptoms } \\
\hline painless swelling & 21 & 44.70 \\
\hline scrotal drop pain & 21 & 44.70 \\
\hline urinary tract irritation & 4 & 8.50 \\
\hline scrotal skin ulceration & 1 & 2.10 \\
\hline \multicolumn{3}{|l|}{ Systemic symptoms } \\
\hline yes & 6 & 12.80 \\
\hline no & 41 & 84.20 \\
\hline \multicolumn{3}{|l|}{ Physical examination } \\
\hline epididymal beaded enlargement & 12 & 25.50 \\
\hline testicular mass & 1 & 2.10 \\
\hline scrotal tenderness & 7 & 14.90 \\
\hline ill-defined epididymal testicular border & 21 & 44.70 \\
\hline sinus formation & 6 & 12.80 \\
\hline \multicolumn{3}{|l|}{ History of tuberculosis } \\
\hline pulmonary tuberculosis & 3 & 0.06 \\
\hline renal tuberculosis & 4 & 0.08 \\
\hline prostate tuberculosis & 1 & 0.02 \\
\hline no & 39 & 0.84 \\
\hline \multicolumn{3}{|l|}{ Preoperative diagnosis } \\
\hline tuberculosis & 41 & 87.20 \\
\hline tumors & 3 & 6.40 \\
\hline masses & 3 & 6.40 \\
\hline
\end{tabular}


Table 2 (on next page)

Table 2

Association between surgical methods and physical examination 


\begin{tabular}{lccc}
\hline & total & $\begin{array}{c}\text { epididymal } \\
\text { resection }\end{array}$ & $\begin{array}{c}\text { epididymis- } \\
\text { testicular resection }\end{array}$ \\
\hline $\begin{array}{l}\text { ill-defined } \quad \text { epididymal } \\
\text { border } \\
\text { epididymal beaded enlargement }\end{array}$ & 21 & $16(76.2 \%)$ & $5(23.8 \%)$ \\
scrotal tenderness & 7 & $10(83.3 \%)$ & $2(16.7 \%)$ \\
sinus formation & 6 & $3(100 \%)$ & 0 \\
testicular mass & 1 & $1(100 \%)$ & $3(100 \%)$ \\
\hline
\end{tabular}

1 
Figure 1

Microscopy images of caseous necrosis

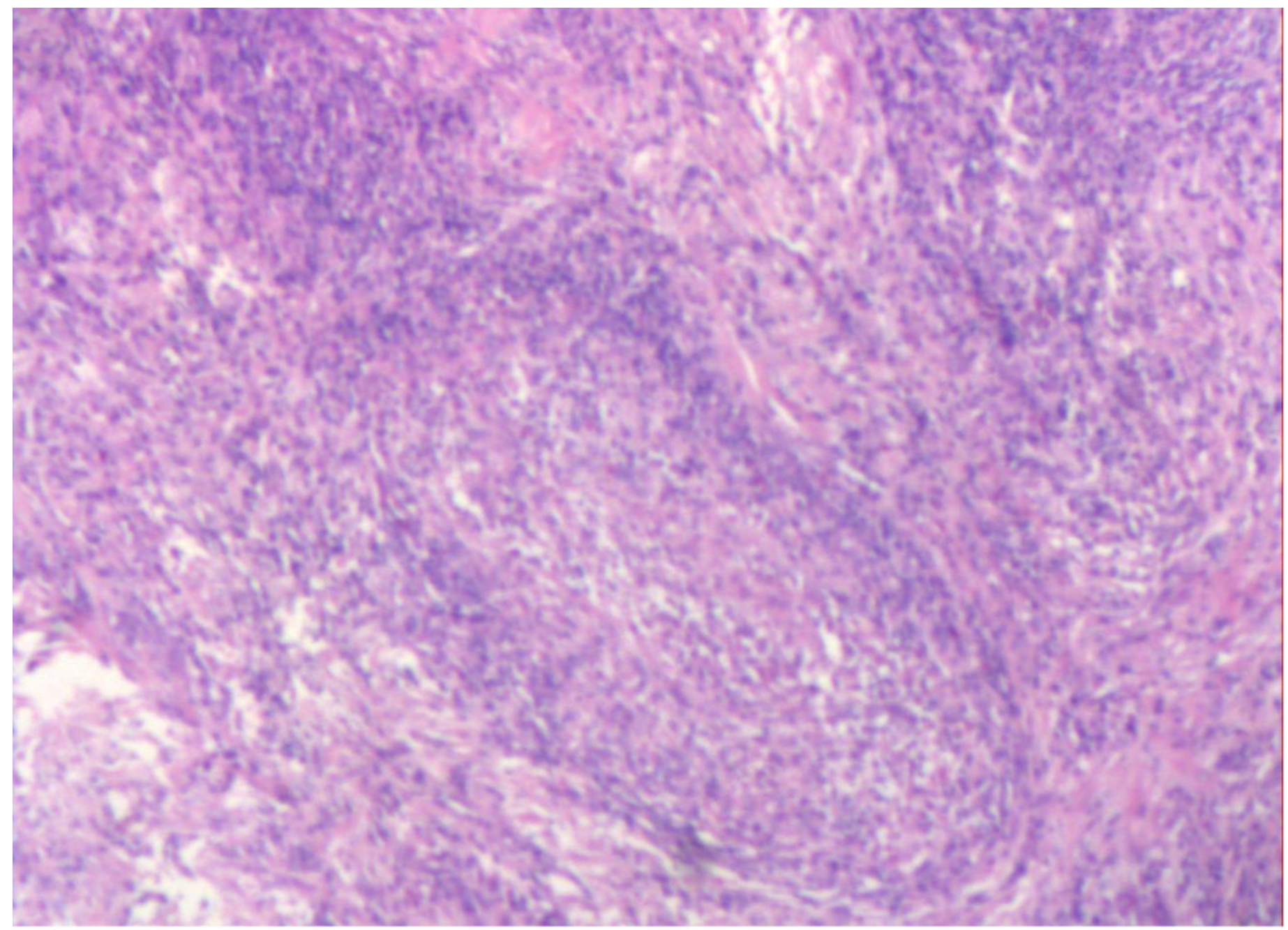


Figure 2

Microscopy images of caseous necrosis

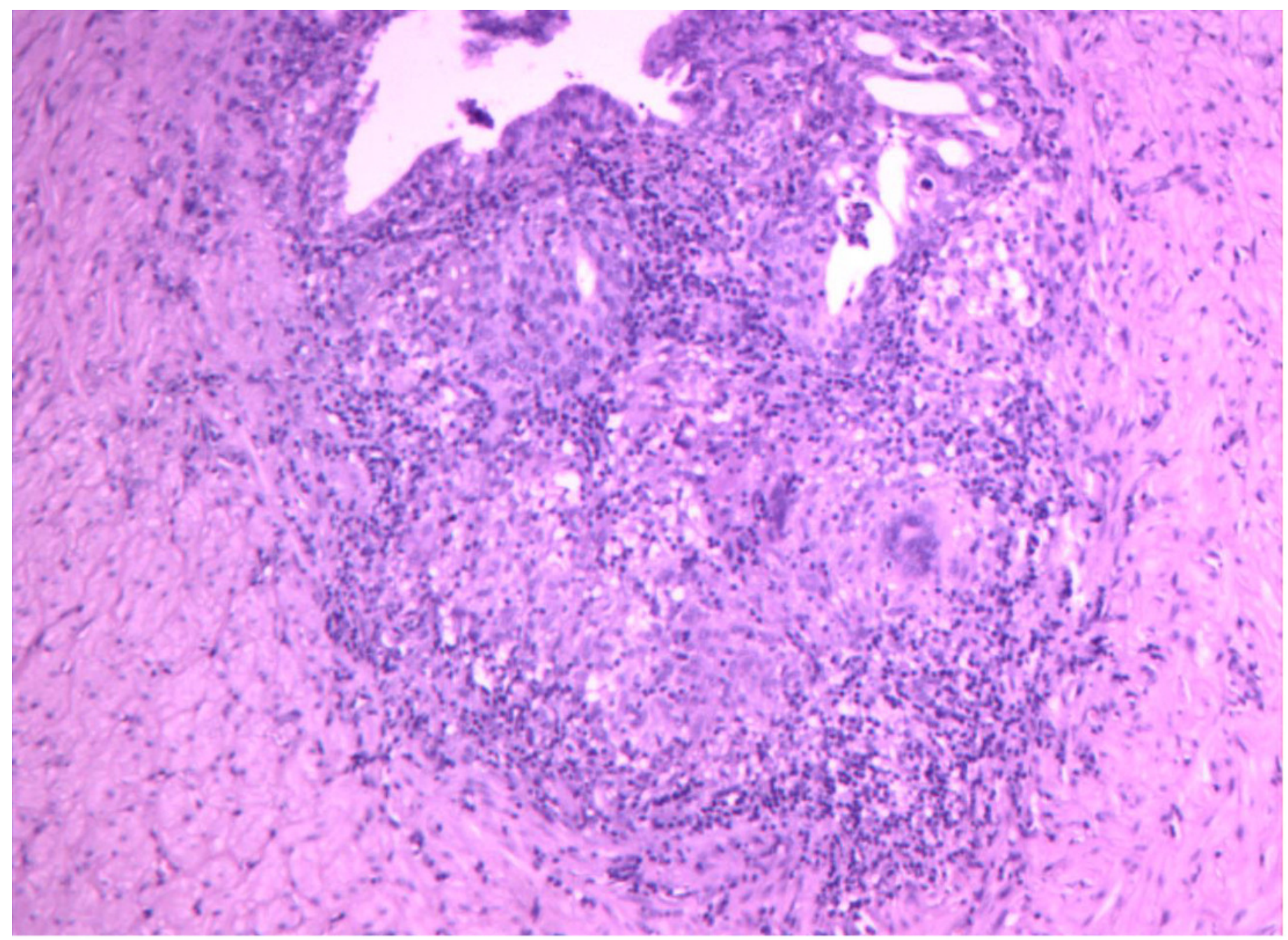

